\title{
Electrocardiographic Changes after Spontaneous Intracerebral Hemorrhage
}

\author{
Pranshu Bhargava' Rahul Singh² \\ ${ }^{1}$ Department of Neurosurgery, Kailash Hospital, Dehradun, \\ Uttrakhand, India \\ ${ }^{2}$ Department of Neurosurgery, Krishna Hospital, Haldwani, \\ Uttrakhand, India
}

\begin{abstract}
Address for correspondence Pranshu Bhargava, MCh, Department of Neurosurgery, Kailash Hospital, Haridwar Road, Dehradun 248001, Uttrakhand, India (e-mail: bhargava_pranshu@rediffmail.com).
\end{abstract}

Indian J Neurosurg 2019;8:16-19

\begin{abstract}
Introduction Electrocardiographic (ECG) changes after ischemic stroke are well documented. However, ECG changes after spontaneous intracerebral hemorrhage ( $\mathrm{SICH}$ ) have been infrequently studied. In many cases, changes occur due to neurogenic causes and may be misinterpreted as primary cardiac events.

Aims This study was undertaken to study ECG changes after SICH.

Materials and Method This study included all SICH patients admitted over 1 year. Patients with intraventricular and subarachnoid hemorrhage without cerebral bleed were excluded, and those with a doubtful history of head injury were also excluded. Management was guided as per standard protocol. All patients were monitored in neurosurgical intensive care unit (ICU). ECG changes were noted and analyzed.

Results Total $47 \mathrm{SICH}$ patients were admitted over 1 year; $0.66 \%$ patients were found to have one or more ECG abnormalities at time of admission. A prolonged QTC interval was the most common finding (39\%), followed by sinus tachycardia or bradycardia and ST-segment changes. All patients with brainstem or intraventricular bleed had changes in ECG, whereas $62 \%$ had basal ganglia (BG) bleed, $43 \%$ thalamic bleed, and $57 \%$ had cortical bleeds had ECG changes, and $0.13 \%$ of patients had concomitant echocardiographic $(\mathrm{ECHO})$ changes also. The most common change in BG lesion was $\mathrm{T}$ inversion as well as QTc prolongation in thalamic lesions. An equal percentage of males and females had ECG changes; however, $80 \%$ of all patients were males. All patients younger than 40 years had changes on ECG, and $75 \%$ of patients in the age group of 61 to 70 were found to have changes on ECG. In one patient with intraventricular hemorrhagic, global hypokinesia was reversed after insertion of ventricular drain and normalization of intracranial pressure (ICP). Although the management of the cardiac events may remain the same, in cases in which the primary cause is neurogenic,

Keywords

- electrocardiographic

- intracranial

- hemorrhage

treating this cause or lowering ICP may help in reverting ECG changes.

Conclusion ECG changes occurring after SICH need to be cautiously interpreted, as the primary cause may be neurogenic and subsequent treatment would require lowering of ICP either surgically or medically.
\end{abstract}

\section{Introduction}

Electrocardiographic (ECG) changes after ischemic stroke and subarachnoid hemorrhage are well documented. ${ }^{1}$ Nonmyocardial causes of ECG changes have also been well documented. ${ }^{2}$ However, ECG changes after spontaneous intracerebral hemorrhage (SICH) have been infrequently studied. In many cases, the changes that occur due to neurogenic causes may be misinterpreted as primary cardiac events. The management will then be

(C)2019 Neurological Surgeons'

Society of India
License terms

()(1) $\Theta \circledast$ 
directed toward cardiac causes rather than medical or surgical reduction in intracranial pressure (ICP). Various studies have also shown a relationship between presence of neurogenic ECG changes and mortality. ${ }^{3,4}$ This study is hence undertaken to study ECG changes after SICH. This will help in timely interventions to reduce ICP and may also help in patient prognostication.

\section{Materials and Method}

This study was conducted at a tertiary care center, prospectively over 1 year. Patients with SICH (supra- and infratentorial) were included. Exclusion criteria were subarachnoid or intraventricular hemorrhage (IVH) without parenchyma bleed, doubtful traumatic bleeds, and patients on anticoagulation. A detailed history and examination was done. All patients were admitted to neurosurgical intensive care unit (ICU) and managed as per standard protocol. All patients had and ECG at time of admission and were monitored continuously. ECG changes occurring were recorded by critical care team and confirmed by the senior consultant. Echocardiography (ECHO) was done in all patients. All changes detected on monitors were confirmed using a 12-lead ECG. The

Table 1 Age and sex distribution of cases

\begin{tabular}{|l|l|l|l|}
\hline Age (y) & M & F & \\
\hline$<40$ & 3 & 0 & 3 \\
\hline $41-50$ & 11 & 0 & 11 \\
\hline $51-60$ & 12 & 4 & 16 \\
\hline $61-70$ & 8 & 4 & 12 \\
\hline$>70$ & 4 & 1 & 5 \\
\hline & 38 & 9 & 47 \\
\hline
\end{tabular}

cardiologist was consulted initially and as per clinical requirement. Neurosurgical management was aimed at lowering of ICP and guided by clinical status in terms of surgery or conservative treatment. The ECG changes were tabulated corresponding to different cerebral areas based on findings of computed tomographic (CT) scan. CT was interpreted jointly by a neurosurgeon and radiologist.

\section{Results}

Total $47 \mathrm{SICH}$ patients were admitted to the neurosurgical ICU over 1 year. In this study, $80.2 \%$ of the patients were males and $93.6 \%$ of patients were older than 40 years. At the time of admission, one or more ECG abnormalities were seen in $66 \%$ of the patients ( - Table 1 ). The most common finding was a prolonged QTc interval (39\%), followed by sinus tachycardia or bradycardia and ST-segment changes (-Fig. 1). All patients with brainstem and intraventricular bleed had changes in ECG, whereas $62 \%$ had basal ganglia (BG) bleed, $43 \%$ thalamic bleeds, and $57 \%$ had cortical bleeds had ECG changes (-Table 2). Concomitant ECHO changes were seen in $13 \%$ of patients (thalamic 2 , IVH 1 , and brainstem 1). One patient had global hypokinesia, one patient had a right wall motion defect, and left ventricular hypertrophy was seen in three patients. The most common change in BG lesion was T-wave inversion as well as QTC prolongation in thalamic lesions ( - Table 3 ). ECG changes were seen in an equal percentage in males and females (-Table 1). ECG changes were seen in all patients younger than 40 years whereas $75 \%$ of patients in the age group of 61 to 70 were found to have changes on ECG (-Table 4). Changes regarding side of bleed were not analyzed. Correlation with mortality could not be done as few patients were shifted to other centers. In one IVH patient, global hypokinesia was reversed after insertion of ventricular drain and normalization of ICP.

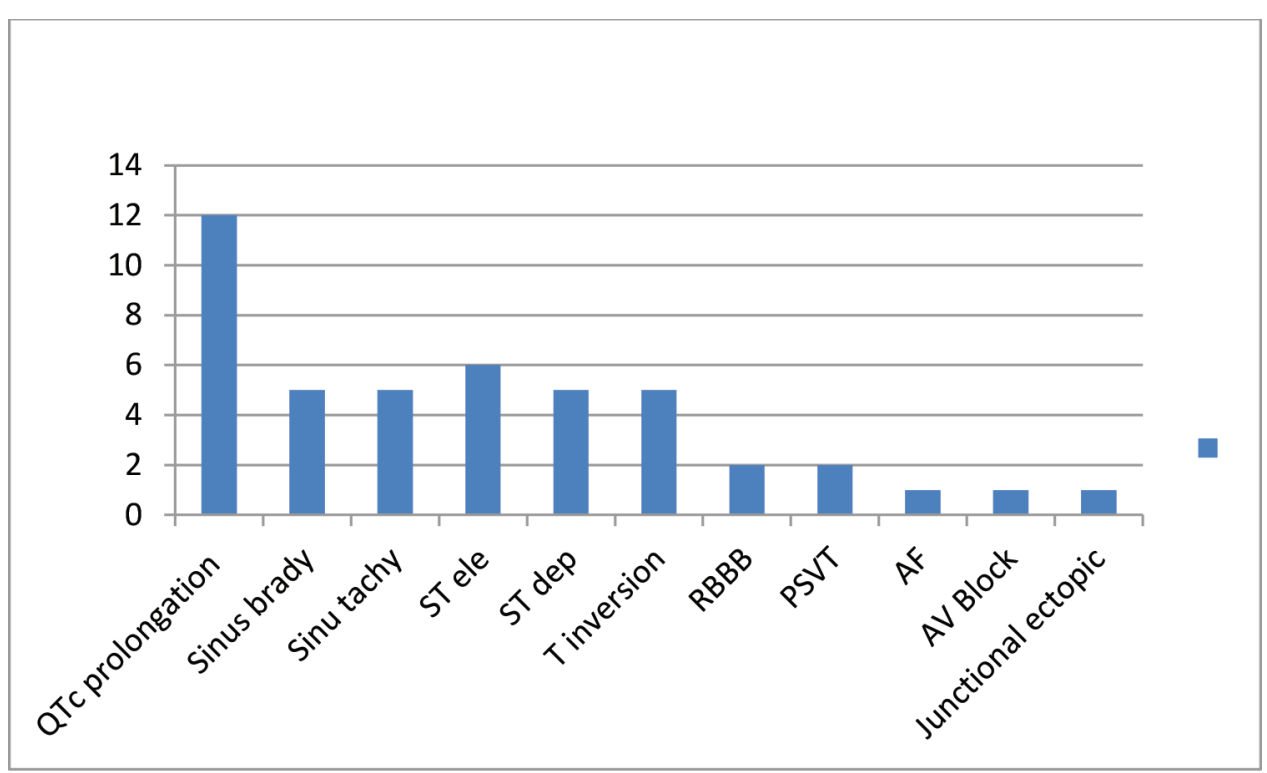

Fig. 1 Distribution of ECG changes. AF, Atrial fibrillation; AV, atrioventricular; PSVT, paroxysmal supraventricular tachycardia; RBBB, right bundle branch block 
Table 2 Percentage distribution of electrocardiographic changes

\begin{tabular}{|l|l|l|l|l|}
\hline Region & Change & No & & $\%$ \\
\hline Thalamus (Th) & 3 & 4 & 7 & 43 \\
\hline Basal ganglia (BG) & 13 & 8 & 21 & 62 \\
\hline Frontal & 2 & 2 & 4 & 50 \\
\hline Temporal & 0 & 0 & 0 & 0 \\
\hline Parietooccipital & 2 & 1 & 3 & 66 \\
\hline Intraventricular hemorrhage (IVH) with bleed & 2 & 0 & 2 & 100 \\
\hline Brainstem & 4 & 0 & 4 & 100 \\
\hline Subarachnoid hemorrhage (SAH) & 4 & 1 & 5 & 80 \\
\hline Cortical venous thrombosis (CVT) with bleed & 1 & 0 & 1 & 100 \\
\hline & 31 & 16 & 47 & \\
\hline
\end{tabular}

Table 3 Region-wise distribution of electrocardiographic changes

\begin{tabular}{|c|c|c|c|c|c|c|c|}
\hline & BG & Th & Cortical & SAH & IVH & Brainstem & CVT with bleed \\
\hline QTc prolonged & 3 & 4 & 1 & 3 & 0 & 1 & 1 \\
\hline Sinus brady & 2 & 1 & 1 & 1 & 0 & 0 & \\
\hline Sinus tachy & 2 & 1 & 0 & 0 & 1 & 1 & \\
\hline ST elevation & 2 & 1 & 1 & 0 & 0 & 2 & \\
\hline ST depression & 2 & 1 & 1 & 0 & 0 & 1 & \\
\hline T inversion & 4 & 0 & 0 & 0 & 0 & 1 & \\
\hline RBBB & 0 & 0 & 1 & 0 & 1 & & \\
\hline PSVT & 1 & 0 & 1 & 0 & 0 & & \\
\hline $\mathrm{AF}$ & 1 & 0 & 0 & 0 & 0 & & \\
\hline AV block & 0 & 1 & 0 & 0 & 0 & & \\
\hline Junctional ectopic & 0 & 1 & 0 & 0 & 0 & & \\
\hline
\end{tabular}

Abbreviations: AF, Atrial fibrillation; AV, atrioventricular; BG, basal ganglia; CVT, cortical venous thrombosis; IVH, intraventricular hemorrhage; PSVT, paroxysmal supraventricular tachycardia; RBBB, right bundle branch block; SAH, subarachnoid hemorrhage; Th, thalamus.

Table 4 Distribution of cases with ECG changes

\begin{tabular}{|l|l|l|l|l|}
\hline Age $(y)$ & M & F & & Percentage \\
\hline$<40$ & 3 & 0 & 3 & 100 \\
\hline $41-50$ & 6 & 0 & 6 & 54 \\
\hline $51-60$ & 8 & 2 & 10 & 62 \\
\hline $61-70$ & 6 & 3 & 9 & 75 \\
\hline$>70$ & 2 & 1 & 3 & 60 \\
\hline & 25 & 6 & 31 & 66 \\
\hline
\end{tabular}

\section{Discussion}

Electrocardiographic changes after ischemic stroke have been well documented. ${ }^{1,2}$ However, ECG changes occurring after SICH have been infrequently studied. In many cases, the changes that occur due to neurogenic causes may be misinterpreted as primary cardiac events. ${ }^{3-5}$ The authors found that a prolonged QTC interval was the most common abnormality seen in SICH patients. This was in accordance with other studies. Similar to other studies, BG and thalamic bleeds had a higher frequency of ECG changes. ${ }^{3-5}$ There are various studies describing the pathophysiology of cardiac events after SICH. It has been proposed that a cardiac cortical rhythm control site is probably present within the middle cerebral artery territory or in the anterior cingulate cortex. ${ }^{6,7}$ Vascular damage to these areas has been postulated to cause cardiac arrhythmias due to a disinhibition of the right insular cortex with resulting increased sympathetic tone. Ischemic involvement of the right hemisphere induces a higher risk for cardiac arrhythmia occurrence than that of the left hemisphere. The autonomic nervous system receives neural input from various parts of the cerebral cortex, hypothalamus, and brainstem, which is extensively interconnected. Derangements of autonomic function have also been shown to be responsible for the disturbances of rate, rhythm, and conduction. ${ }^{6-8}$ 
Tachycardia and pressor responses were more commonly seen after stimulation of the right insular cortex and after experimental stimulation of the left vagus that innervates the atrioventricular node and the cardiac conduction system. Bradycardia was found to be more common after stimulation of the left insular cortex or the right vagus nerve that innervates the sinoatrial node. ${ }^{8}$ It has been demonstrated that ECG changes were present in more than $90 \%$ of unselected patients with ischemic stroke and intracerebral hemorrhage; however, the prevalence was much lower after exclusion of patients with preexisting heart disease. QT abnormalities have been associated with a higher in-hospital mortality. ${ }^{9,10}$

QT prolongation after SICH was also found to be an independent risk factor for subsequent stroke besides traditional risk factors. ${ }^{11}$ Popescu et al have demonstrated that ECG changes are frequent in hemorrhagic stroke and correlate with greater in-hospital mortality. They found that QTc and ST-segment abnormalities were the only two factors associated with poor outcome. ${ }^{4}$

\section{Conclusion}

Electrocardiographic changes occurring after SICH need to be cautiously interpreted, as the primary cause may be neurogenic and subsequent treatment would require lowering of ICP either surgically or medically, rather than managing the changes as a primary cardiac event.

\section{Conflict of Interest}

None.

\section{References}

1 Cropp GJ, Manning GW. Electrocardiographic changes simulating myocardial ischemia and infarction associated with spontaneous intracranial hemorrhage. Circulation 1960;22:25-38

2 Dimant J, Grob D. Electrocardiographic changes and myocardial damage in patients with acute cerebrovascular accidents. Stroke 1977;8(4):448-455

3 Liu Q, Ding Y, Yan P, Zhang JH, Lei H. Electrocardiographic abnormalities in patients with intracerebral hemorrhage. Acta Neurochir Suppl (Wien) 2011;111:353-356

4 Popescu D, Laza C, Mergeani A, Bajenaru OA, Antochi FA. Lead electrocardiogram changes after supratentorial intracerebral hemorrhage. Maedica (Buchar) 2012;7(4):290-294

5 Jaikar SKB, Divya NS, Rajan C. Analysis of electrocardiographic changes in cerebrovascular accidents. IOSR J Dental Med Sci 2014;13(5):25-29

6 Daniele O, Caravaglios G, Fierro B, Natalè E. Stroke and cardiac arrhythmias. J Stroke Cerebrovasc Dis 2002;11(1):28-33

7 Critchley HD, Mathias CJ, Josephs O, et al. Human cingulate cortex and autonomic control: converging neuroimaging and clinical evidence. Brain 2003;126(Pt 10):2139-2152

8 Keller C, Williams A. Cardiac dysrhythmias associated with central nervous system dysfunction. J Neurosci Nurs 1993;25(6):349-355

9 Gölbaşi Z, Selçoki Y, Eraslan T, Kaya D, Aydoğdu S. QT dispersion. Is it an independent risk factor for in-hospital mortality in patients with intracerebral hemorrhage? Jpn Heart J 1999;40(4):405-411

10 Pickham D, Helfenbein E, Shinn JA, et al. High prevalence of corrected QT interval prolongation in acutely ill patients is associated with mortality: results of the QT in Practice (QTIP) study. Crit Care Med 2012;40(2):394-399

11 Soliman EZ, Howard G, Cushman M, et al. Prolongation of QTc and risk of stroke: the REGARDS (REasons for Geographic and Racial Differences in Stroke) study. J Am Coll Cardiol 2012;59(16):1460-1467 